\title{
APRI score non-invasive marker of metabolic syndrome in breast carcinoma patients
}

\author{
S.K. Verma ${ }^{\mathrm{a}}$, Nidhi Kaeley ${ }^{\mathrm{b}, *}$, Sohaib Ahmad ${ }^{\mathrm{c}}$, Minakshi Dhar ${ }^{\mathrm{d}}$, Abhimav Chhabra ${ }^{\mathrm{e}}$ \\ a Oncology Department, Himalayan Institute Of Medical Sciences, Dehradun, India \\ ${ }^{\mathrm{b}}$ Emergency Medicine, AIIMS Rishikesh, India \\ ${ }^{c}$ Medicine, HIHT Dehradun, India \\ d General Medicine, AIIMS Rishikesh, India \\ e Himalayan Institute Of Medical Sciences, Dehradun, India
}

A R T I C L E I N F O

\section{Keywords:}

Breast carcinoma

Metabolic syndrome

BMI

Post-menopausal women

\begin{abstract}
A B S T R A C T
Background: Breast carcinoma is one of the commonest cancer in women accounting for major health burden worldwide. Metabolic syndrome is a known risk factor of non-alcoholic steatohepatitis (NASH) as well as breast carcinoma. APRI (Aspartate aminotransferase to platelet ratio index), a known non-invasive marker of liver fibrosis and steatohepatitis has never been studied in patients of metabolic syndrome with breast carcinoma. Breast carcinoma has various modifiable risk factors such as obesity and alcohol consumption. Hence, we compared APRI score in patients with breast carcinoma in order to establish a possible correlation.

Aims and Objectives: Our primary objective was to study APRI (Aspartate aminotransferase to platelet ratio index) score in patients of breast carcinoma with and without metabolic syndrome. The secondary objective was to study APRI score in post-menopausal women with breast carcinoma.

Material and Method: This prospective observational study included 151 patients of breast carcinoma, these patients were sub grouped into two, with and without metabolic syndrome and pre and post-menopausal women. Multiple demographic and biochemical parameters including APRI score were studied in these groups. The sensitivity and specificity of APRI score was calculated in these groups.

Results: A total of 151 patients of breast carcinoma were included in the study group.53.64\% patients with breast carcinoma had metabolic syndrome. The mean values of AST (Aspartate aminotransferase), BMI (Body mass index), FBS (Fasting blood sugar) and APRI score were significantly higher in the patients with metabolic syndrome. More than $50 \%$ patients of breast carcinoma belonged to the post-menopausal age group. Mean values of AST (aspartate aminotransferase), BMI (body mass index), FBS (fasting blood sugar) and APRI were higher in this group. The area under the receiver operating characteristics (ROC) curve of APRI score of metabolic syndrome patients was 0.93 and that of post-menopausal women was 0.82 .

Conclusion: APRI score can be used as a surrogate marker of metabolic syndrome in patients with breast carcinoma. It is also useful in planning preventive strategies in patients with breast carcinoma especially in postmenopausal age group.
\end{abstract}

\section{Introduction}

The most common cancer in women worldwide is breast carcinoma. As per 2012 epidemiological data, around 1.7 million new cases of breast cancer were added to the existing cases. ${ }^{1}$

The 'National Cancer Institute' has recognized factors like overweight, lack of physical activity and alcohol consumption as preventable risk factors of breast carcinoma. Many of these risk factors are components of metabolic syndrome. ${ }^{2,3}$ According to NCEP ATP III guidelines (National cholesterol education programme adult treatment panel III), criteria of metabolic syndrome includes three or more of the following (3)

1 Abdominal obesity (waist circumference $>35$ in. in women)

2 Triglyceride $>150 \mathrm{mg} / \mathrm{dl}$

3 HDL-c (high density lipoprotein - cholesterol $<50 \mathrm{mg} / \mathrm{dl}$ )

$4 \mathrm{BP}$ (blood pressure) $>130 / 85 \mathrm{mmHg}$

5 Fasting blood sugar $>110 \mathrm{mg} / \mathrm{dl}^{3,4}$

One of the important cause of NAFLD (non-alcoholic fatty liver

\footnotetext{
* Corresponding author

E-mail address: drnidhi_kaeley@yahoo.com (N. Kaeley).
} 
disease) is metabolic syndrome. NASH (non-alcoholic steato-hepatitis) fibrosis score has six variables. They are age, hyperglycemia, BMI (body mass index), platelet count, albumin and AST/ALT (aspartate transaminase to alanine transaminase) ratio. This score includes few components of metabolic syndrome such as hyperglycemia and BMI (body mass index) which could be an indirect marker of abdominal obesity. ${ }^{5}$ APRI score (aspartate transaminase to platelet ratio) has been approved by a previous study as a non-invasive marker of NAFLD. ${ }^{6}$ NAFLD is a manifestation of metabolic syndrome, suggesting a possible link between APRI score and presence of metabolic syndrome.

In our study, we attempted to establish a possible relationship between APRI score and metabolic syndrome in the patients with breast carcinoma. Various epidemiological studies in the past have demonstrated obesity as a risk factor of breast carcinoma especially in postmenopausal women. ${ }^{7-9}$ There are multiple drugs in the market used for the treatment of breast carcinoma in post-menopausal women like exemestane and tamoxifen. Side effects of these drugs include hyperlipidemia and reduced bone mineral density. ${ }^{10}$ Hepatopathy in the form of fatty liver which has been reported with tamoxifen. ${ }^{11}$ Keeping this observation in mind, we compared APRI score in post and pre-menopausal women of breast carcinoma as a direct marker of liver injury and indirect marker of metabolic syndrome.

\section{Material and methods}

This prospective observational study included 151 diagnosed cases of breast carcinoma attending oncology outpatient department and admitted in oncology wards over a period of one year (2015-2016), in a tertiary care hospital of Uttarakhand. All the patients were grouped as 1) with and without metabolic syndrome 2) post and pre-menopausal women.

The demographic and biochemical parameters including APRI score of all the patients of breast carcinoma were compiled and tabulated. APRI (Apartate aminotranferase to platelet ratio index) was calculated as

$$
A P R I=\frac{\text { ASTlevel }(I U / L)}{\text { AST upper limit of normal }}
$$

(AST upper limit of normal) $=38 \mathrm{IU} / \mathrm{L}$

Diagnosis of breast carcinoma was based on the evaluation of triple assessment technique utilizing radiological, histopathological/ cytological and clinical criteriae. Metabolic syndrome was diagnosed using NCEP-ATP III criteriae. ${ }^{3}$

We compared ALT (alanine transaminase), AST (aspartate transaminase), FBS (fasting blood sugar), BMI (body mass index) and APRI score in all the groups, mean value of all the above mentioned parameters were compared using unpaired ' $\mathrm{t}$ ' test and ROC curve. ROC Curve was used for calculating the sensitivity and specificity of APRI score in patients with metabolic syndrome and post-menopausal women with breast carcinoma.

Data analysis was done using SPSS (Statistical Package for Social Sciences) version 20.0 statistical analysis software. $P$ value less than 0.05 was associated with statistical significance association.

\section{Results}

A total of 151 diagnosed cases of breast carcinoma were included in the study. They were further subdivided as with and without metabolic syndrome and pre and post-menopausal syndrome. As evident in Table 1, the mean age of the patients with metabolic syndrome was $51 \pm 10.89$ years, more than that of without metabolic syndrome. Mean values of AST (aspartate transaminase), albumin levels, BMI (body mass index), FBS (fasting blood sugar) and APRI were significantly higher in patients of breast carcinoma with metabolic
Table 1

Biochemical profile of patients of breast carcinoma with and without metabolic syndrome.

\begin{tabular}{lllc}
\hline Parameters & $\begin{array}{l}\text { With metabolic } \\
\text { syndrome } \\
(\mathrm{n}=81,53.04 \%)\end{array}$ & $\begin{array}{l}\text { Without metabolic } \\
\text { syndrome }(\mathrm{n}=70, \\
46.35 \%)\end{array}$ & P value \\
\hline Age (yrs) & $51.00 \pm 10.89$ & $45.96 \pm 12.18$ & $<0.05$ \\
ALT (IU/L) & $36.16 \pm 41.90$ & $45.96 \pm 12.18$ & 0.191 \\
AST (IU/L) & $66.59 \pm 58.36$ & $31.97 \pm 15.91$ & $<0.05$ \\
Albumin (mg/ & $3.59 \pm 0.48$ & $3.83 \pm 0.36$ & $<0.05$ \\
$\quad \mathrm{dl})$ & & $22.41 \pm 3.95$ & $<0.05$ \\
BMI $\left(\mathrm{kg} / \mathrm{m}^{2}\right)$ & $27.58 \pm 3.64$ & $94.16 \pm 24.56$ & $<0.05$ \\
FBS $(\mathrm{mg} / \mathrm{dl})$ & $143.44 \pm 48.00$ & $0.32 \pm 0.14$ & $<0.05$ \\
APRI & $0.96 \pm 0.74$ & & \\
\hline
\end{tabular}

Table 2

Biochemical profile of patients of breast carcinoma with and without postmenopausal syndrome.

\begin{tabular}{llll}
\hline Parameters & $\begin{array}{l}\text { Post-menopausal } \\
(\mathrm{n}=94,62.25 \%)\end{array}$ & $\begin{array}{l}\text { Pre-menopausal }(\mathrm{n}=57, \\
37.74 \%)\end{array}$ & P value \\
& & & \\
\hline Age (yrs) & $53.54 \pm 10.31$ & $40.61 \pm 9.31$ & $<0.05$ \\
ALT (IU/L) & $36.00 \pm 39.58$ & $27.28 \pm 24.51$ & $<0.05$ \\
AST (IU/L) & $62.41 \pm 55.33$ & $30.96 \pm 16.45$ & $<0.05$ \\
Albumin (mg/ & $3.62 \pm 0.46$ & $3.85 \pm 0.39$ & $<0.05$ \\
$\quad \mathrm{dl})$ & & & $<0.05$ \\
BMI (kg/m $\left.{ }^{2}\right)$ & $27.18 \pm 3.93$ & $22.51 \pm 3.63$ & $<0.05$ \\
FBS (mg/dl) & $134.12 \pm 49.64$ & $98.3 \pm 27.53$ & $<0.05$ \\
APRI & $0.87 \pm 0.71$ & $0.36 \pm 0.27$ & \\
\hline
\end{tabular}

syndrome and in post-menopausal age group (Table 2). More than $50 \%$ of the patients of breast carcinoma were from urban background and were literate. Ultrasonography of abdomen was done in 130 patients of breast carcinoma, 85 patients had abnormal findings, most commonly fatty liver. Out of these 85 patients, 70 patients had metabolic syndrome. The abnormal liver findings were not confirmed on liver biopsy.

The area under the receiver operating characteristics (ROC) curve of APRI for metabolic syndrome patients was 0.93 with a cut off of 0.25 showing sensitivity of $98.8 \%$ and specificity of $60 \%$ (Fig. 1).

As shown in Fig. 2 the ROC curve of APRI score for post-menopausal women showed area of 0.82 with cut off 0.25 showing sensitivity of

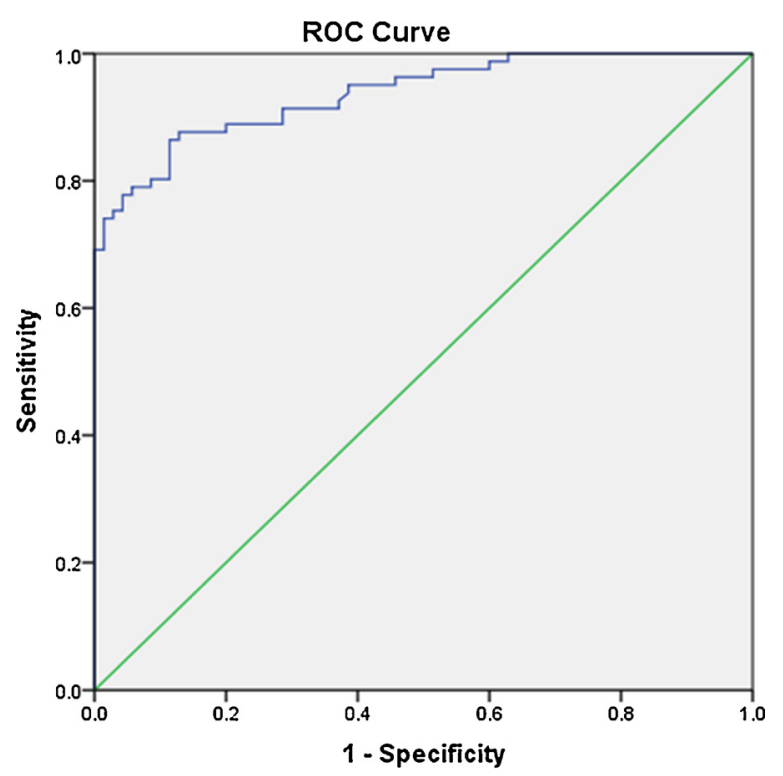

Diagonal segments are produced by ties.

Fig. 1. Area Under the Curve of ROC curve of breast carcinoma patients with metabolic syndrome. 


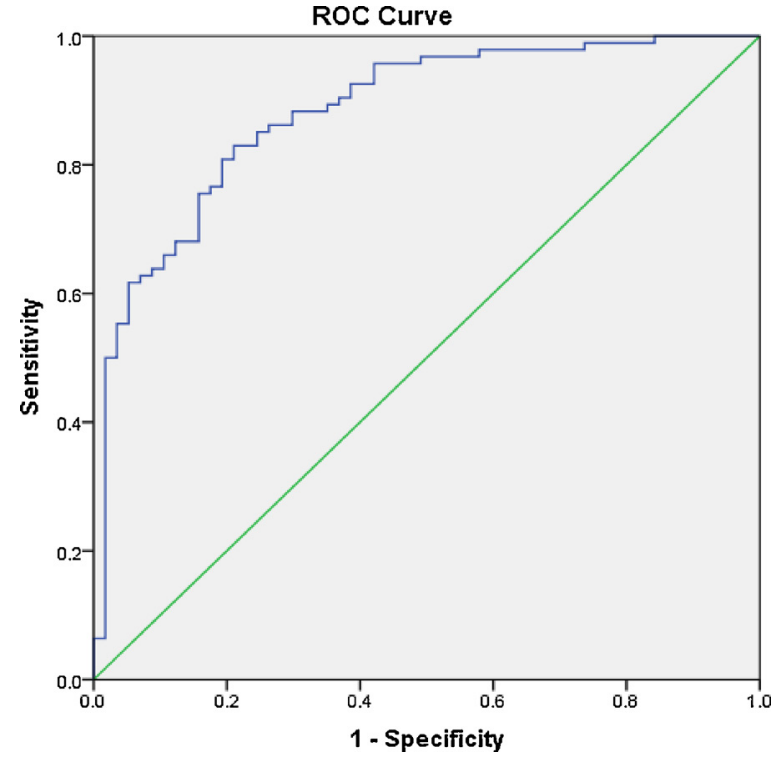

Fig. 2. Area Under the Curve FIG2-ROC CURVE OFAPRI SCORE IN POST MENOPAUSAL WOMEN OF BREAST CARCINOMA.

$96.8 \%$ and specificity of $52.6 \%$.

\section{Discussion}

Metabolic syndrome is a potential risk factor of breast carcinoma. ${ }^{3}$ It is a key causative factor for the development of non-alcoholic steatohepatitis. ${ }^{12}$ Also drugs like tamoxifen with prolonged usage of more than six months in patients of breast carcinoma lead to the development of non-alcoholic fatty liver disease. ${ }^{13-16}$

APRI score (Aspartate aminotransferase to platelet ratio index) has been considered as a non-invasive marker for the diagnosis of non-alcoholic steatohepatitis. $^{6}$

In our study, we compared APRI score in patient of breast carcinoma with and without metabolic syndrome. Mean value of APRI score in patients of breast carcinoma with metabolic syndrome was significantly higher than that in patients without metabolic syndrome. Thus, APRI score can be used as a surrogate marker of metabolic syndrome in patients with breast carcinoma.

The incidence of breast carcinoma was higher in post-menopausal age group (62.25\%). More than $50 \%$ of these women belonged to urban background and were literate. This result was consistent with the studies conducted in India and United states of America. The reason for increased prevalence of metabolic syndrome in women with urban background could be due to rising rates of obesity among urban population. The lack of exercise and high fat diet are the contributing factors. ${ }^{17}$

Majority of the post-menopausal women attained menopause after the age of 45 years. Mean age of post-menopausal women with breast carcinoma in our study was $53.84 \pm 10.31$ years. It has been observed that higher age of menopause is associated with increased risk of breast carcinoma by almost $3 \%$ with each year older at menopause. ${ }^{17,18} \mathrm{Me}-$ tabolic syndrome is associated with $52 \%$ increased risk of post-menopausal breast carcinoma. ${ }^{19}$ In our study $62.25 \%$ of post-menopausal women had breast carcinoma and more than $50 \%$ of these patients had metabolic syndrome. In the recent past, several theories have explained the relationship of metabolic syndrome and breast carcinoma. Estrogen levels are higher in obese post-menopausal women which indirectly increase the levels of circulating estradiol by decreasing the fraction of sex hormone binding globulin (SHBG). ${ }^{20}$ Risk of insulin resistance and metabolic syndrome increases with lower levels of SHBG. ${ }^{21,22}$

Two adipokines named leptin and adipnectin affect breast carcinoma pathogenesis. ${ }^{23}$ Obesity, insulin resistance and metabolic syndrome are important causes of increased leptin levels. ${ }^{24}$ Leptin acts as an antagonist to adiponectin. Breast cancer cell lines are stimulated by leptin whereas adipnectin inhibits them. ${ }^{23}$ Obesity reduces adipnectin levels. ${ }^{23}$ Multiple mechanisms have been proposed explaining the mitogenic effect of insulin on breast cancer cells like by stimulating action of estradiol and lowering production of SHBG. ${ }^{26,27}$

The prevalence of breast carcinoma as well as metabolic syndrome is on the rise. They have common risk factors. Metabolic syndrome has been found to influence breast carcinoma via multiple interrelated signaling pathways involving insulin, cytokines, estrogen and growth factors. ${ }^{28}$ It has been interesting to note that hypercholesterolemia has been a major risk factor for the estrogen receptor positive breast carcinoma patients. It leads to retarded response to endocrinal therapies. 27-hydroxycholesterol is responsible for the growth of estrogen receptor dependent and liver-X-receptor dependent metastasis in mouse models of breast carcinoma. ${ }^{29}$ Hence lowering the cholesterol levels is a useful strategy in both prevention and treatment of breast carcinoma. Considering this relationship between metabolic syndrome and breast carcinoma, APRI score can be used as an assessment tool to screen patients of breast carcinoma with metabolic syndrome.

The authors did not have any competing interests for the study. No special funding was provided by institute.

Dr Skverma has contributed in conception or design of data, drafting the article and data interpretation. Dr Nidhi Kaeley has contributed in data designing, drafting framework of the article and interpretation of results. DrMinakshi Dhar, DrAbhinav Chhabra and Dr Sohaib Ahmad have also contributed significantly in framing the article.

\section{Conclusion}

APRI score, a tool used for assessing NASH can serve as an indirect marker of metabolic syndrome, especially in post-menopausal women of breast carcinoma. This can assist health care providers in formulating primary and secondary preventive measures of breast carcinoma.

The highlights of our study include1) Significantly fair number of patients of breast carcinoma were included in the studt.2) No previous study has studied APRI score in patients of breast carcinoma with and without metabolic syndrome.3) APRI score can be used as a screening measure to find risk factors of breast carcinoma especially in younger population.

The shortcomings of our study include1) We did not compare APRI score in patients with and without breast carcinoma.2) The ultrasound should have been done in all the patients of breast carcinoma to find appropriate co-relation of presence of metabolic syndrome in breast carcinoma with ultrasound findings.3) The presence of fatty liver could have been proved by doing liver biopsy.

This is a new concept. More such studies are needed in this direction.

\section{References}

1. Ferlay J, Soerjomataram I, Ervik M, et al. GLOBOCAN 2012 v1.0, cancer incidence and mortality worldwide: IARC cancerBase No. 11. Lyon, France: International Agency for Research on Cancer; 2013.

2. National Cancer Institute. Breast cancer-risk factors. http://www.cancer.gov/ cancertopics/wyntk/breast/page4.

3. Grundy SM, Brewer Jr HB, Cleeman JI, Smith Jr SC, Lenfant C. Definition of metabolic syndrome: report of the National Heart, Lung, and Blood Institute/American Heart Association conference on scientific issues related to definition. Circulation. 2004;109(3):433-438.

4. Third report of the National Cholesterol Education Program (NCEP) expert panel on detection, evaluation, and treatment of high blood cholesterol in adults (adult treatment panel III) final report. Circulation. 2002;106(25):3143-3421.

5. Angulo P, Hui JM, Marchesini G, et al. The NAFLD fibrosis score: a non-invasive system that identifies liver fibrosis in patients with NAFLD. Hepatology. 2007:45:846-854.

6. Kruger FC, Daniels CR, Kidd M, et al. APRI: a simple bedside marker for advanced 
fibrosis that can avoid liver biopsy in patients with NAFLD/NASH. S Afr Med J. 2011;101:477-480.

7. Rinaldi S, Key TJ, Peeters PH, et al. Anthropometric measures, endogenous sex steroids and breast cancer risk in postmenopausal women: a study within the EPIC cohort. Int J Cancer. 2006;118(no. 11):2832-2839.

8. Tehard B, Lahmann PH, Riboli E, Clavel-Chapelon F. Anthropometry, breast cancer and menopausal status: use of repeated measurements over 10 years of followup-results of the French E3N women's cohort study. Int J Cancer. 2004;111(no. 2):264-269.

9. Van Den Brandt PA, Spiegelman D, Yaun SS, et al. Pooled analysis of prospective cohort studies on height, weight, and breast cancer risk. Am J Epidemiol. 2000;152(no. 6):514-527.

10. Hozumil Y, Suemasu K, Takei H, et al. The effect of exemestane, anastrozole, and tamoxifen on lipid profiles in Japanese postmenopausal early breast cancer patients: final results of National Surgical Adjuvant Study BC 04, the TEAM Japan sub-study. Ann Oncol. 2011;22(No. 8 (August)):1777-1782.

11. Lin $\mathrm{Y}$, Liu J, Zhang X, et al. A prospective, randomized study on hepatotoxicity of anastrozole compared with tamoxifen in women with breast cancer. Cancer Sci. 2014;105:1182-1188.

12. Andrade GC, Fujise LH, de Filho JE, Oliveira F. Non-alcoholic fatty liver disease (NAFLD) in different populations: a clinical and epidemiological study - sample of São José do Rio Preto. Rev Assoc Med Braz. 2016;62(3):218-226.

13. Pratt DS, Knox TA, Erban J. Tamoxifen-induced steatohepatitis. Ann Intern Med. 1995; 123(3):236

14. Struben VM, Hespenheide EE, Caldwell SH. Nonalcoholic steatohepatitis and cryptogenic cirrhosis within kindreds. Am J Med. 2000;108(1):9-13.

15. Genuth S, Alberti KG, Bennett P, et al. Follow-up report on the diagnosis of diabetes mellitus. Diabetes Care. 2003;26(11):3160-3167.

16. Van der Valk M, Bisschop PH, Romijn JA, et al. Lipodystrophy in HIV-1 positive patients is associated with insulin resistance in multiple metabolic pathways. AIDS. 2001;15(16):2093-2100.
17. Sandhu DS, Sandhu S, Karwasra RK, Marwah S. Profile of breast cancer patients at a tertiary care hospital in North India. Indian J Cancer. 2010;47:16-22.

18. McCormack VA, dos Santos Silva I. Breast density and parenchymal patterns as markers of breast cancer risk: a meta-analysis. Cancer Epidemiol Biomarkers Prev. 2006;15:1159-1169.

19. Esposito K, Chiodini P, Capuano A, et al. Metabolic syndrome and postmenopausal breast cancer: systematic review and meta-analysis. Menopause. 2013;20(12 (December)):1301-1309.

20. Stephenson GD, Rose DP. Breast cancer and obesity: an update. Nutr Cancer. 2003;45(no. 1):1-16.

21. Gruen DG, Connor EB. Sex hormone-binding globulin and glucose tolerance in postmenopausal women: the Rancho Bernardo study. Diabetes Care. 1997;20(no. 4):645-649.

22. Sherif K, Kushne HR, Falkner BE. Sex hormone-binding globulin and insulin resistance in African-American women. Metabolism. 1998;47(no. 1):70-74.

23. Rose P, Komninou D, Stephenson GD. Obesity, adipocytokines, and insulin resistance in breast cancer. Obes Rev. 2004;5(no. 3):153-165.

24. Davis LV, et al. Adiposity, type 2 diabetes and the metabolic syndrome in breast cancer. Obes Rev. 2007;8(no. 5):395-408.

26. Panno ML, Salerno M, Pezzi V, et al. Effect of oestradiol and insulin on the proliferative pattern and on oestrogen and progesterone receptor contents in MCF-7 cells. J Cancer Res Clin Oncol. 1996;122(no. 12):745-749.

27. Plymate SR, Matej LA, Jones RE, Friedl KE. Inhibition of sex hormone-binding globulin production in the human Hepatoma (Hep G2) cell line by insulin and prolactin. $J$ Clin Endocrinol Metab. 1988;67(no. 3):460-464.

28. Ruixing Y, Qiming F, Dezhai Y, et al. Comparison of demography, diet, lifestyle, and serum lipid levels between the Guangxi Bai Ku Yao and Han populations. J Lipid Res. 2007;48(12 (December)):2673-2681.

29. Riccardi G, Rivellese AA. Dietary treatment of the metabolic syndrome-the optimal diet. Br J Nutr. 2000;83(Suppl 1):S143-S148. 